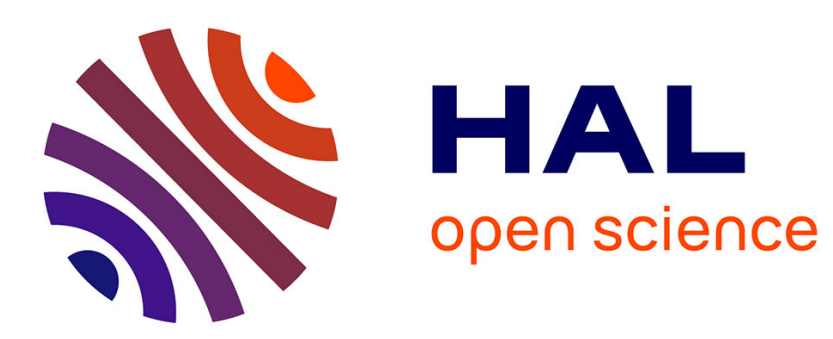

\title{
Heat convection within evaporating droplets in strong aerodynamic interactions
}

Guillaume Castanet, Bruno Frackowiak, Cameron Tropea, Fabrice Lemoine

\section{To cite this version:}

Guillaume Castanet, Bruno Frackowiak, Cameron Tropea, Fabrice Lemoine. Heat convection within evaporating droplets in strong aerodynamic interactions. International Journal of Heat and Mass Transfer, 2011, 54 (15-16), pp.3267-3276. 10.1016/j.ijheatmasstransfer.2011.03.060 . hal-01499162

\section{HAL Id: hal-01499162 \\ https://hal.univ-lorraine.fr/hal-01499162}

Submitted on 31 Mar 2017

HAL is a multi-disciplinary open access archive for the deposit and dissemination of scientific research documents, whether they are published or not. The documents may come from teaching and research institutions in France or abroad, or from public or private research centers.
L'archive ouverte pluridisciplinaire HAL, est destinée au dépôt et à la diffusion de documents scientifiques de niveau recherche, publiés ou non, émanant des établissements d'enseignement et de recherche français ou étrangers, des laboratoires publics ou privés.

\section{(ㅇ)(1) $\$$}

Distributed under a Creative Commons Attribution - NonCommercial - NoDerivatives| 4.0 


\title{
Heat convection within evaporating droplets in strong aerodynamic interactions
}

\author{
Guillaume Castanet $^{\mathrm{a}, *}$, Bruno Frackowiak $^{\mathrm{b}}$, Cameron Tropea ${ }^{\mathrm{b}}$, Fabrice Lemoine ${ }^{\mathrm{a}}$ \\ ${ }^{a}$ LEMTA, Nancy Université, CNRS, 54504 Vandoeuvre-lès-Nancy, France \\ ${ }^{\mathrm{b}}$ Institute of Fluid Mechanics and Aerodynamics, Center of Smart Interfaces, Technische Universität Darmstadt, D-64287 Darmstadt, Germany
}

\section{Keywords:}

Spray

Evaporation

Droplet

Combustion

LIF

Marangoni effect

\begin{abstract}
A B S T R A C T
The temperature field within evaporating ethanol droplets is investigated, relying on the two-color laser induced fluorescence (LIF) measurement technique and on a Direct Numerical Simulation (DNS). The configuration studied corresponds to a monodisperse droplet stream in a diffusion flame sustained by the droplet vapor. An experimental probe volume, small compared to the droplet size, is used to characterize the temperature field within the droplets, whereas DNS takes into account key aspects of the droplet heating and evaporation such as the non-uniform and transient stress, and the mass and heat transfer coefficients at the droplet surface. These investigations reveal that the frictional stresses are strongly reduced due to the small spacing between the droplets. They also show that the Marangoni effect has a significant influence on the internal motion and hence on the internal temperature field.
\end{abstract}

\section{Introduction}

In an Euler-Lagrange approach, numerical simulations of droplet laden flows require heat and mass transfer models for the droplet through sources terms in the gas phase, usually based on correlations obtained from numerical or experimental studies. Although the case of an isolated droplet has received considerable attention throughout the decades [1-6], this is not directly applicable to droplets in dense sprays that experience strong aerodynamic interactions. When the distance between the droplets becomes the same order as their size, droplets undergo not only a strong reduction of their aerodynamic drag force, but also a significant decrease in their heating and evaporation rates.

Interactions between closely spaced droplets have been numerically investigated by several authors. The point source method was used by Labowsky [7] and Marberry et al. [8] to describe the steady evaporation of finite motionless arrays of droplets containing up to 20 droplets. More sophisticated simulations, but with fewer droplets, were performed by Brzustowski et al. [9] or by Chiang and Sirignano $[10,11]$. The latter were able to account for several aspects of the droplet vaporization: the forced convection of the gas phase, the transient deceleration due to the drag force, the diameter decrease, the respective motion between the droplets, the liquid phase internal circulation, the transient heating of the liquid phase. However, the limited number of droplets involved in those simulations do not realistically capture the interactions

\footnotetext{
* Corresponding author. Tel.: +33 3839957 32; fax: +33 383999944 .

E-mail address: Guillaume.Castanet@ensem.inpl-nancy.fr (G. Castanet).
}

occurring in a dense spray. On the experimental side, investigations have been carried out on simplified configurations, mainly around the monodisperse droplet stream [12-22], where a liquid jet is disintegrated into a line of periodic droplets, exploiting excited Rayleigh breakup. Virepinte et al. [13] and Atthasit et al. [14] suggested a correction that should be applied to the drag coefficient and the vaporization rate of the droplets in combustion to take into account the interactions. This correction was formulated as a function of the spacing parameter $C$, which is defined as the distance between the droplets $S_{d}$ divided by their diameter $D_{d}$. Similar corrections were also proposed by Castanet et al. [18] for the Nusselt and Sherwood numbers using the two-color laserinduced fluorescence technique to characterize the droplet temperature evolution.

Nevertheless, difficulties remain in the prediction of the interaction effect on the convection within the liquid phase. This is especially a problem in applications where the fuel droplets are directly injected inside the combustion chamber and strong unsteady effects can be expected. The duration of the heating phase is generally far from negligible compared to the residence time in the chamber. However, in most Computational Fluid Dynamics (CFD) codes, the heating has been modeled assuming that there is a spherical symmetry for the temperature field inside the droplet $[1,2]$. More accurate but still simplified, the Hill Vortex model relies on a given liquid velocity field depending on the friction coefficient $C_{f}$ to take into account the internal convection [5]. However, models to assess the value of $C_{f}$ in the case of interacting droplets are currently missing, although there is some evidence that it can be strongly reduced compared to an isolated droplet. 


\section{Nomenclature}

Greek symbols

$\epsilon \quad$ difference between experimental and theoretical fields

[1]

$\lambda \quad$ thermal conductivity $\mathrm{W} \mathrm{m}^{-1} \mathrm{~K}^{-1}$

$\lambda_{c} \quad$ center wavelength of the optical filter $\mathrm{nm}$

$\mu \quad$ dynamic viscosity $\mathrm{N} \mathrm{s} \mathrm{m}^{-2}$

$v_{d} \quad$ part of the droplet volume $\mathrm{m}^{3}$

$\theta \quad$ angle (spherical coordinates) rad

$\rho \quad$ density $\mathrm{kg} \mathrm{m}^{-3}$

$\sigma \quad$ surface tension $\mathrm{J} \mathrm{m}^{-2}$

$\tau_{p r} \quad$ normal viscous stress $\mathrm{N} \mathrm{m}^{-2}$

$\tau_{p \theta} \quad$ tangential viscous stress $\mathrm{N} \mathrm{m}^{-2}$

$\xi \quad$ normalized temperature [1]

Latin symbols

$a_{l} \quad$ thermal diffusivity $\mathrm{m}^{2} \mathrm{~s}^{-1}$

C spacing parameter [1]

$\mathrm{Cd} \quad$ drag coefficient [1]

$C_{f} \quad$ friction coefficient [1]

$D \quad$ mass diffusion coefficient $\mathrm{m}^{2} \mathrm{~s}^{-1}$

$D_{d} \quad$ droplet diameter $\mathrm{m}$

$D_{T} \quad$ Soret thermal diffusion coefficient $\mathrm{kg} \mathrm{m}^{-1} \mathrm{~s}^{-1}$

$f \quad$ frequency of the droplet disintegration $\mathrm{s}^{-1}$

$F_{\text {drag }} \quad$ non-dimensional total drag $\mathrm{N} \mathrm{m}^{-2}$

$F_{\text {form }}$ non-dimensional form drag $\mathrm{N} \mathrm{m}^{-2}$

$F_{\text {friction }}$ non-dimensional friction drag $\mathrm{N} \mathrm{m}^{-2}$

$F_{\text {Marangoni }}$ non-dimensional Marangoni force $\mathrm{N} \mathrm{m}^{-2}$
Fthrust non-dimensional thrust force $\mathrm{N} \mathrm{m}^{-2}$

FWHM full width at half maximum (of the optical filter) nm

$L_{v} \quad$ latent heat $\mathrm{J} \mathrm{kg}^{-1}$

$\dot{m} \quad$ surface flow rate $\mathrm{kg} \mathrm{s}^{-1} \mathrm{~m}^{-2}$

$P \quad$ pressure $\mathrm{Pa}$

Pe $\quad$ Peclet number [1]

$Q_{l_{\text {effective }}}$ effective heat flux $\mathrm{W} \mathrm{m}^{-2}$

$q_{m} \quad$ total flow rate $\mathrm{kg} \mathrm{s}^{-1}$

$r \quad$ radius $m$

$S \quad$ droplet surface $\mathrm{m}^{2}$

$S_{d} \quad$ distance between two consecutive droplets $\mathrm{m}$

$T$ temperature $\mathrm{K}$

$U_{S} \quad$ liquid maximal velocity $\mathrm{m} \mathrm{s}^{-1}$

$V_{d} \quad$ droplet velocity in the stream $\mathrm{m} \mathrm{s}^{-1}$

$V_{e} \quad$ vapor ejection velocity $\mathrm{m} \mathrm{s}^{-1}$

$V_{\theta} \quad$ tangential velocity $\mathrm{m} \mathrm{s}^{-1}$

$V_{r} \quad$ radial velocity $\mathrm{m} \mathrm{s}^{-1}$

$x, y, z \quad$ Cartesian coordinates $\mathrm{m}$

$Y \quad$ mass fraction [1]

Indices

gas relating to the gas phase

interface relating to the liquid-gas interface

iso relating to the isolated droplet case

liq relating to the liquid phase

$s \quad$ relating to the droplet surface

vap relating to the evaporated specie
Measurements of the temperature distribution within combusting ethanol droplets in interaction have been achieved by Castanet et al. $[15,19]$ and compared to numerical simulations based on the Hill vortex approach, leading to an estimate of the coefficient $C_{f}$. For a spacing parameter $C$ in the range 3 to 5 , they found that the $C_{f}$ can be decreased by about $90 \%$ [19]. Nevertheless, their approach suffers from some limitations related to the Hill vortex model. For instance, the transitional phase in the liquid motion, as well as the non-uniform temperature and transfer parameters at the droplet surface were not considered. These drawbacks could be avoided with Direct Numerical Simulation (DNS), making this approach particularly attractive for the analysis of the internal heat convection.

Numerical simulations carried out by Dwyer and co-workers [6], solving the complete Navier Stokes equations in the gas and liquid phase for the isolated droplet case, have pointed out the significance of the Marangoni effect on the liquid motion. This phenomenon results from the dependence of the surface tension on the temperature, which creates an additional stress to the viscous one, accelerating or slowing down the liquid motion. More recently, Frackowiak et al. [23] managed to simulate the case of a monodisperse droplet stream with DNS by imposing periodic conditions at the limits of the simulation domain containing two consecutive droplets. During the droplets cooling, Frackowiak et al. [23] noticed that the Marangoni stress can be more significant than the viscous stress, splitting the liquid motion into two contra rotating vortices.

The present study is complementary to these previous investigations since it aims at comparing temperature fields obtained experimentally by two-color laser-induced fluorescence $[15,19]$ and the DNS approach developed by Frackowiak et al. [23]. The computational procedure and the experimental setup will be described first, followed by a detailed analysis based on two experimental cases of ethanol combusting droplets. Special attention will be paid to the Marangoni effect on the internal motion and the temperature distributions.

As in $[15,19]$, the size of the droplet in this study is in the range of $100-200 \mu \mathrm{m}$. This is large in comparison to the size of droplets injected in an internal combustion (IC) engine, but experimentally smaller droplets are difficult to investigate in such detail. Nevertheless, the higher injection velocity in an automotive spray maintains an internal droplet convection at a level that is rather comparable with this study.

\section{Experimental setup}

A linear monodisperse droplet stream is generated by Rayleigh disintegration of a liquid jet undergoing a mechanical vibration provided by a piezoceramic. The voltage applied on the piezoceramic is a square wave, whose amplitude influences the position of the break-up zone. For certain resonant frequencies $f$, the liquid jet breaks up into equally spaced and monosized droplets. The liquid flows through an orifice with a diameter of $100 \mu \mathrm{m}$, and its temperature is regulated in the injector body with the help of an external heated water circulation, measured with a thermocouple just before the injector exit. An electrically heated coil, placed just after the break-up of the liquid jet is used to ignite a laminar diffusion flame surrounding the droplet stream (Fig. 1). The droplet velocity $V_{d}$ is measured by a Laser Doppler Anemometry (LDA) system and distance from the injector exit can be converted into time according to this velocity. The diameter of the droplets just after the break-up is determined from the injected volumetric liquid flow rate, knowing the frequency of the droplet disintegration $f$. Since $S_{d}=\frac{V_{d}}{f}$, the dimensionless spacing parameter $C=\frac{S_{d}}{D_{d}}$ is also determined from the measurement of the droplet size and velocity.

The possibility of mapping the temperature within droplets with the two-color laser induced fluorescence technique (2cLIF) 


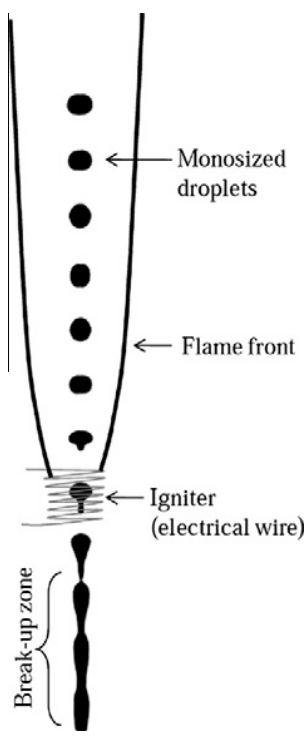

Fig. 1. Monodisperse droplet stream in combustion.

has been reported in previous papers $[15,19]$. The fuel is seeded with a low concentrated fluorescent dye $\left(10^{-6} \mathrm{~mol} / \mathrm{L}\right)$, presently pyrromethene 597-C8, provided by Exciton [24]. The choice of this dye relies on the high temperature dependence of its fluorescence spectrum and its high solubility in ethanol. The properties of pyrromethene 597-8C9 as a fluorescence tracer for measuring the temperature with the two-color laser-induced fluorescence technique has been extensively described in [16]. For most of its components, the optical setup used in this experiment is similar to that presented in this article. It differs mainly by the focal length of the lenses placed in front of the detection and emission probes, which define the measurement volume. A schematic view of the optical layout is depicted in Fig. 2.

The fluorescence is induced by the green line of an argon ion laser ( $514.5 \mathrm{~nm}$ ). Two laser beams crossing at $30^{\circ}$ in the $y-z$ plane are used for the excitation of the fluorescence (Fig. 3). The detection optics is positioned perpendicularly to the droplet trajectory and focused on the beam intersection. With this arrangement of the laser beams, the positioning of the detection probe in the $z$ direction is easier compared to a single beam: when the system is properly aligned, the intersection of the beams appears in the center of the image formed by the lenses of the detection probe.

The light scattered at the wavelength of the laser is eliminated with the use of a notch filter (Super Notch Plus, Kayser Optical, optical density 6). The fluorescence signal emitted within the measurement volume is transmitted by an optical fiber to a set

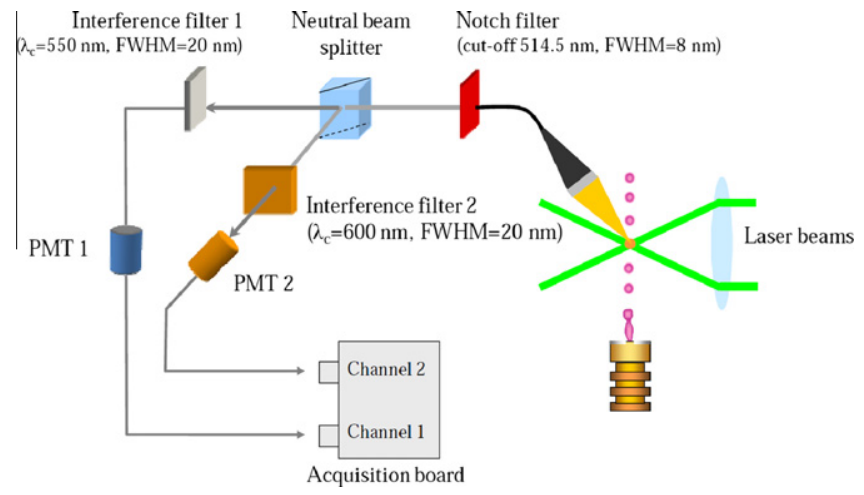

Fig. 2. Fluorescence detection setup.

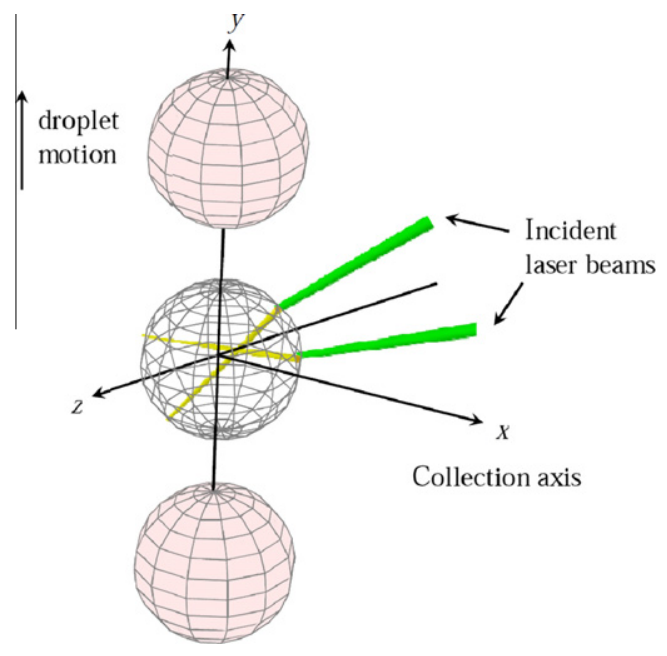

Fig. 3. Optical layout.

of beamsplitters and optical filters (Omega Optical, 3RD Millennium Bandpass filters) which allows the division the fluorescence signal in two spectral bands: the 1 st band with $\lambda_{c}=550 \mathrm{~nm}, F W H M=$ $20 \mathrm{~nm}$ and the $2 \mathrm{nd}$ band with $\lambda_{c}=600 \mathrm{~nm}, F W H M=20 \mathrm{~nm}$. These two spectral bands present highly different temperature sensitivities, as explained in the previous paper [16]. The ratio of the fluorescence intensity measured on these two spectral bands is function of the temperature and the fluorescence dependencies on tracer concentration, probe volume dimensions, laser intensity and optical layout are removed [16]. A calibration at a reference temperature yields the correspondance between the local temperature and the measured fluorescence ratio [16]. Furthermore, the non-linear effect of the diameter recently highlighted by Labergue et al. [22] is not significant for the droplet diameters considered in this study, especially when using pyrromethene 597-C8 as a fluorescence tracer, since the fluorescence ratio is affected for droplet sizes below $110 \mu \mathrm{m}$ with this tracer. Finally, the photon flux is converted into an electrical signal using a pair of photomultiplier tubes (Hamamatsu photosensors modules H9307-04) equipped with fastresponding preamplifiers and signal acquisition is performed at a sampling rate of $10 \mathrm{MHz}$. The accuracy of the temperature measurement is about $1{ }^{\circ} \mathrm{C}$, taking into account the bias due to the statistical convergence of the measurement data and the errors related to the calibration.

If the probe volume is larger than the droplet, a volume averaged droplet temperature is obtained [16]. Conversely, in order to obtain the temperature field within the droplets, the laser beams are focused to form a measurement volume several times smaller than the droplets. The measurement volume is given by the intersection between the droplet, the laser beams and the solid angle of the collection optics. Presently, the focused laser beams have a beam waist of $20 \mu \mathrm{m}$, while the optical fiber (behind the detection probe in Fig. 3) images a disk of about $34 \mu \mathrm{m}$ in the focal plane of the detection probe. This yields a measurement volume size of about $20 \times 20 \times 34 \mu^{3}$ whereas the droplet size is about $200 \mu \mathrm{m}$ in the experiments. However, this size is only an estimate since it depends slightly on the exact position of the droplet and the optics due to light refraction at the droplet surface.

When the droplets cross the laser beams, different zones of the droplet interior are successively illuminated and a local temperature is obtained. The injector is then shifted progressively by steps of 10 to $20 \mu \mathrm{m}$ in the $x$ direction to explore the droplet internal field. For each $x$ position of the injector, droplets are scanned vertically (along the $y$ axis) as they cross the probe volume formed by the intersection of the laser beams. The fluorescence signal is then 
processed to obtain the time evolution of the temperature. The droplet velocity is measured by LDA which determines accurately the vertical position $y$ of the droplet center at any time of the signal acquisition. One difficulty arises from the refraction of the laser beams at the droplet surface.

Geometrical optics is used to find the exact location of the measurement zone inside the droplet. Knowing the relative position of the droplet center and the laser beams, the distribution of the laser light intensity within the droplet is determined according to ray tracing $[15,19]$. Subsequently, ray tracing is also used to identify the regions inside the droplet that are in the field of view of the detection optics. From these calculations, the contribution of any specific part of the droplet to the total collected fluorescence signal is quantified. The measured temperature is attributed to the points that have the highest contribution to the fluorescence signal and whose total contribution is equal to $20 \%$ of the global signal. The calculations are repeated for about 200 different positions of the droplet regarding the laser beam system and the detection optics. An interpolation between the measurement points obtained for these 200 positions yields the temperature maps that are presented in Section 4. Calculations reveal that the temperature field would be significantly distorted if light refraction is not accounted for. An overview of this effect of beam refraction on the position of the measurement volume is available in [17]. Additionally, the regions close to the surface of the droplet are not strongly illuminated, so their contribution to the fluorescence signal remains limited. For this reason, it is difficult to find a relevant temperature for these regions and it was decided not to assign a temperature to them, as it can be seen in the Figs. 5 and 6 .

\section{Numerical simulation}

The numerical simulation is based on a two-way coupling between the external flow computations with the CFD software OpenFOAM[25], and an in-house code solving the time dependent Navier-Stokes equations for the internal fields of the droplet [23]. The configuration is axisymmetric, and the quasi-static evaporation hypothesis is adopted for evaluating the mass vapor flow rate per unit surface. The interface is also assumed to remain spherical for low Weber numbers. Additionally, the radiative heat transfer, the gravity, the pressure force work, the gas solubility in the liquid phase and the heat resulting from viscous dissipation are neglected. The fluids are assumed to be Newtonian and incompressible. The diameter decrease and the variation in the spacing parameter $C$ are neglected during the simulated period of time. Presently, strong interaction between the droplets will be considered, therefore the drag force and the transfer coefficients will have particularly low values, making the simulation possible over as much as $40 \mathrm{~ms}$ without losing accuracy due to the droplet size reduction (Table 1 ).

Fig. 4 presents a schematic view of the simulation domain. The gas flow is computed around two consecutive droplets in an Eulerian approach. Periodic boundary conditions are set between the inlet and the outlet of the domain to consider the case of a

Table 1

Experimental parameters.

\begin{tabular}{lll}
\hline & Case 1 & Case 2 \\
\hline Injection temperature & $24{ }^{\circ} \mathrm{C}$ & $24{ }^{\circ} \mathrm{C}$ \\
Droplets diameter & $179 \mu \mathrm{m}$ & $233 \mu \mathrm{m}$ \\
Parameter $C=\frac{S_{d}}{D_{d}}$ & 2.3 & 4.0 \\
Ambient temperature & $1000 \mathrm{~K}$ & $1150 \mathrm{~K}$ \\
Droplet velocity & $6.7 \mathrm{~m} / \mathrm{s}$ & $10.1 \mathrm{~m} / \mathrm{s}$ \\
Weber number & 0.1 & 0.3 \\
Diameter decrease after $40 \mathrm{~ms}$ & $6 \%$ & $8 \%$ \\
\hline
\end{tabular}

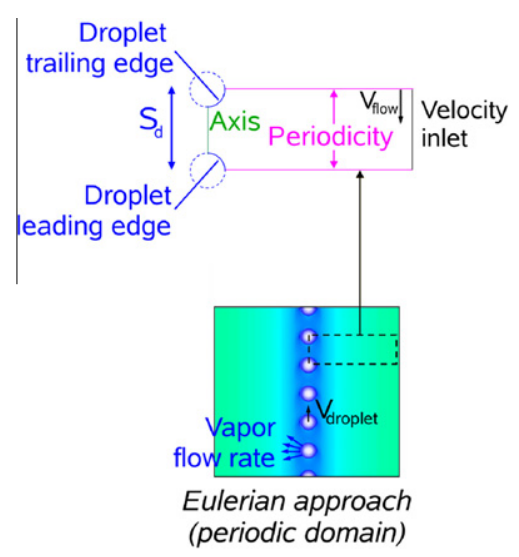

Fig. 4. Schematic view of the simulation domain with the Eulerian approach.

monodisperse droplet stream. With the periodic condition, this configuration is the same as considering a single droplet: the upstream droplet surface corresponds to the trailing edge of this droplet, whereas the downstream droplet surface corresponds to its leading edge.

Although in the experiment droplets are moving in a quiescent environment, the droplet is motionless with this approach, in a flow of axial bulk velocity equal to the experimental value of droplet velocity. Therefore, the far-field boundary conditions correspond to a uniform dry air flow having a velocity equal to the droplet velocity in the experiment. The physical properties are explicitly determined according to the temperature, pressure and species fields.

When an infinite line of droplets is considered, as the periodic condition implies, there is normally no stationary solution because there is no limitation for the radial diffusion of the temperature and vapor concentration. In contrast, for a semi-infinite droplet stream, a stationary solution exists. The steadiness is reached when the entrained air counteracts the vapor diffusion from the droplet surface. In order to simulate the practical case of a semiinfinite jet with a periodic condition, the same procedure as described in [23] is used. On the one hand, the evolution of the boundary layer is simulated at a larger scale by modeling the jet as a semi-infinite cylinder in a Lagrangian approach. On the other hand, successive simulations are performed using the Eulerian periodic domain. Between each simulation, the droplet temperature and velocity fields are reset to their uniform injection values. However, the external flow is not modified. From an initially uniform and dry air flow, simulation after simulation the gas flow evolves due to the temperature, momentum and species diffusion in the radial direction. One simulation is considered as valid when the external flow is close to the Lagrangian simulation around the cylinder. Retroactively, the simulation of the boundary layer with the cylinder is refined by considering the vapor flow rate and temperature calculated around the droplets.

The combustion is not considered directly as a heat source dependent on the droplet evaporation. Instead, as proposed in [20], the combustion is taken into account by imposing a higher temperature to the far field. Measurements of the gas temperature around a monodisperse stream of ethanol droplets in combustion were performed by the ONERA with the help of the CARS. Data are reported by Maqua et al. [20] for different spacing parameters. On the jet axis, the gas temperature was measured around $1000 \mathrm{~K}$ and it is slightly increasing with the droplet spacing. Temperatures in the far field are chosen on the basis of these results.

The Stefan flow and its effects on the heat and mass transfer are taken into account: each droplet is considered as a rigid mass flow source supplying a second species to the surrounding laminar air 
flow. The droplet surface temperature results from the computations of the liquid phase. At the liquid-gas interface, a liquid-vapor equilibrium is assumed. Following Antoine's law, the vapor fraction at the droplet surface is equal to its saturation value calculated from the liquid temperature. The total flow rate between phases depends on the computed vapor mass fraction and the temperature gradients at the droplet surface (Eq. (1)). The effective heat flux used for the computation of the liquid phase can be deduced from the external flow (Eq. (2)).

$$
\begin{aligned}
& \dot{m}=-\frac{1}{1-Y_{\text {vap }, s}}\left[\left.\rho D \frac{d Y_{\text {vap }}}{d r}\right|_{s}+\left.\frac{D_{T_{\text {vap }}}}{T_{s}} \cdot \frac{d T}{d r}\right|_{s}\right] \\
& Q_{\text {leffective }}=\left.\lambda \frac{\partial T}{\partial r}\right|_{s}-L_{v} \cdot \dot{m}
\end{aligned}
$$

At the interface between the liquid and the gas, there is a mechanical equilibrium between the local stresses acting on the liquid and gas sides and on the droplet interface. The projection onto spherical coordinates yields the Eq. (3).

$$
\left\{\begin{array}{l}
\tau_{p r, \text { liq }}-\tau_{\text {pr.gas }}=0 \\
\tau_{p \theta, \text { liq }}-\tau_{p \theta, \text { gas }}-\tau_{p \theta, \text { interface }}=0
\end{array}\right.
$$

Neglecting the tangential gradients in comparison to the radial gradients, as usually assumed in boundary-layer problems, provides the Eq. (4).

$$
\left\{\begin{array}{l}
\tau_{p r, \text { liq }}=\tau_{p r, \text { gas }}=\left.2 \mu \frac{\partial V_{r}}{\partial r}\right|_{s} \\
\tau_{p \theta, \text { liq }}=\tau_{p \theta, \text { gas }}+\tau_{p \theta, \text { interface }}=\left.\mu \frac{\partial V_{\theta}}{\partial r}\right|_{s}+\left.\frac{1}{r_{s}} \cdot \frac{\partial \sigma}{\partial T} \cdot \frac{\partial T}{\partial \theta}\right|_{s}
\end{array}\right.
$$

In Eq. (4), the Marangoni effect [6] is taken into account through the interfacial stress, in order to evaluate the total stress at the droplet surface. Finally, to interpret the simulation results, this stress is integrated over the droplet and the resulting force (divided by the frontal area) is split into several components that can be evaluated separately: the Marangoni force (Eq. (5)), the friction drag (Eq. (6)) and the form drag (Eq. (7)). As usually defined, the total drag corresponds to the sum of the friction and form drag (Eq. (8)), excluding the Marangoni force. Moreover, it is important to note that the thrust force associated with the Stefan flow is neglected in this study. The justification for this simplification will be given later in Section 4.3 for the situations that are considered in this study.

$$
\begin{aligned}
& F_{\text {Marangoni }}=\frac{1}{\pi r_{s}^{2}} \times\left.\iint_{S} \frac{1}{r_{s}} \cdot \frac{\partial \sigma}{\partial T} \cdot \frac{\partial T}{\partial \theta}\right|_{S} \sin \theta d S \\
& F_{\text {friction }}=\frac{1}{\pi r_{S}^{2}} \times \iint_{S}\left(\left.\mu \frac{\partial V_{\theta}}{\partial r}\right|_{S} \sin \theta-\left.2 \mu \frac{\partial V_{r}}{\partial r}\right|_{S} \cos \theta\right) d S=\frac{1}{2} \rho V_{d}^{2} C_{f} \\
& F_{\text {form }}=\frac{1}{\pi r_{S}^{2}} \times \iint_{S} P_{S} \cos \theta d S \\
& F_{\text {drag }}=F_{\text {friction }}+F_{\text {form }}=\frac{1}{2} \rho V_{d}^{2} C d
\end{aligned}
$$

\section{Results and discussion}

\subsection{Description of the droplet temperature field}

Two cases are considered in this study. Their corresponding spacing parameters, droplet diameters and velocities are given in Table 1.

The experimentally measured distributions of the droplet temperature are presented in Figs. 5a and 6a. The domain portrayed in these figures corresponds to a meridian plane. It can be noticed that the region near the droplet surface is excluded from the temperature maps, because the deflection of the laser beams towards the interior of the droplet leads to a weak illumination of this region. In case 1 (Fig. 5a), the isothermal lines appear rather circular and concentric which indicates that heat convection has a very limited influence compared to heat diffusion. Models based on the assumption of a spherical symmetry of the temperature field seem to be adapted to describe the heating of these droplets. The low velocity and spacing of the droplets explain the limited influence of the internal convection on the temperature distribution in this case.

In contrast to this first case, temperature fields corresponding to case 2 (Fig. 6a) exhibit significant differences of temperature between the leading and the trailing edges of the droplets, respectively at the top and the bottom of the temperature maps. Temperature measurements agree qualitatively well with what can be expected from heat convection by a vortex system similar to the spherical Hill vortex solution. The surface in contact with hot air is the first region to be heated. Then, due to internal circulation, heat is transported to the bottom of the droplet and finally it goes up into the core of the droplet. Very similar observations have been already made in a previous paper also related to ethanol
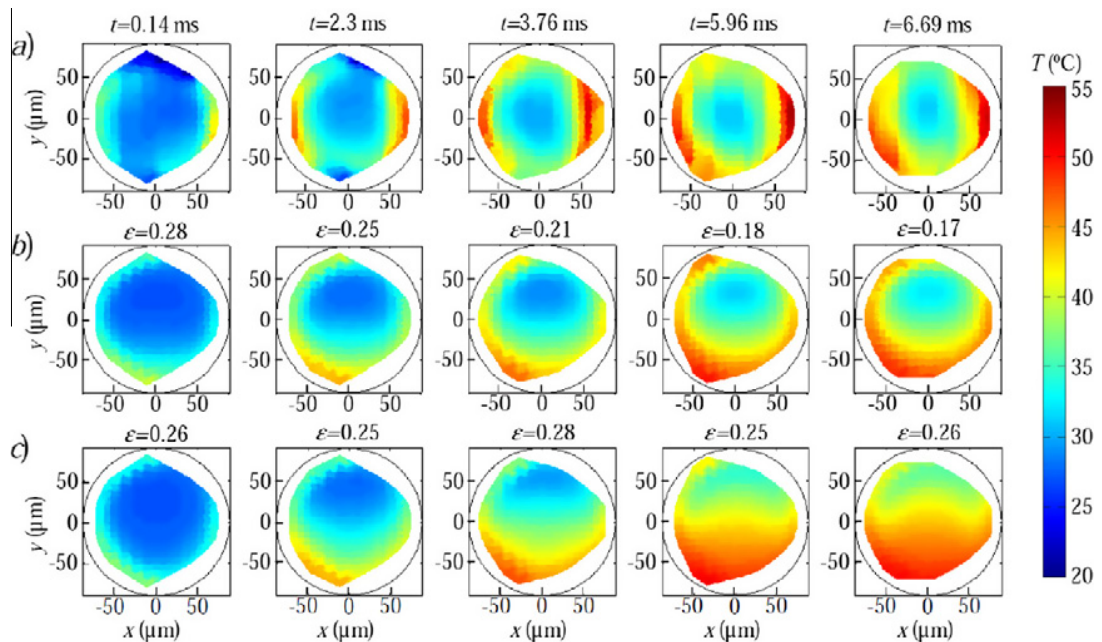

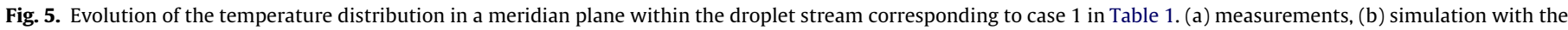
Marangoni effect, (c) simulation without the Marangoni effect, $\epsilon$ : normalized difference between the experimental and the theoretical fields as defined in Eq. (9)). 

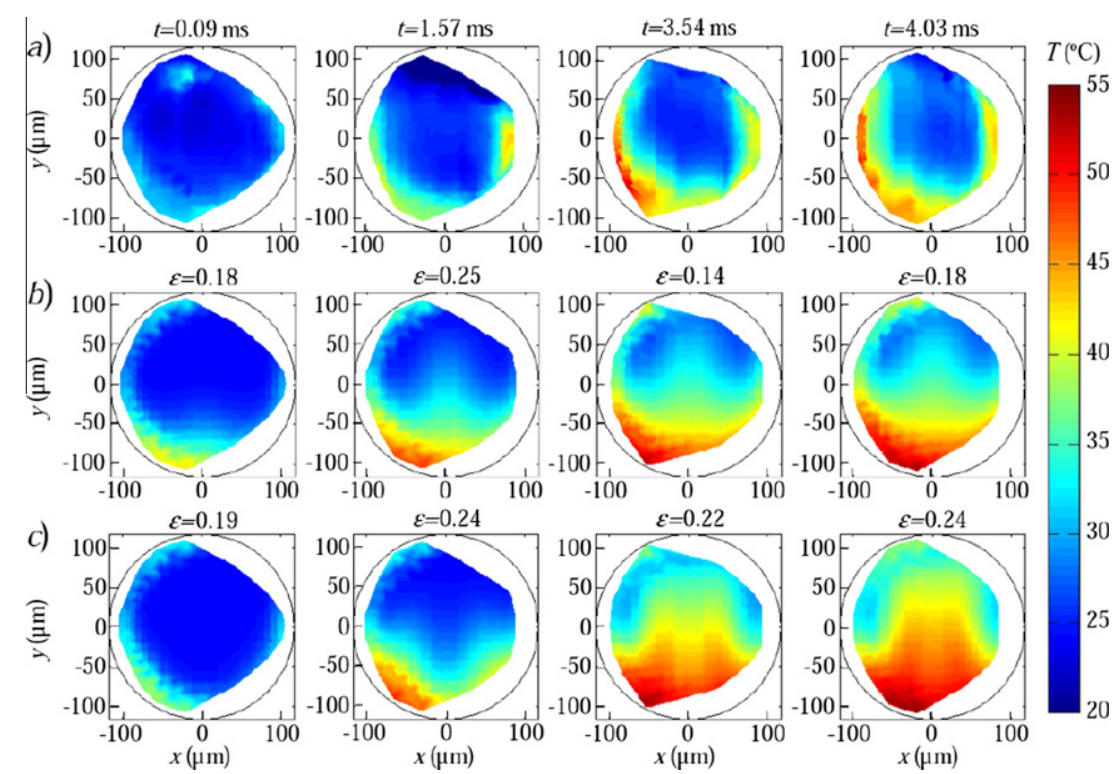

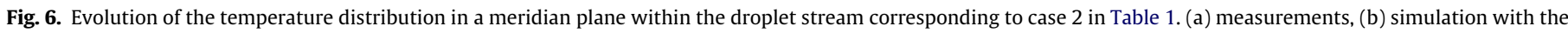
Marangoni effect, (c) simulation without the Marangoni effect, $\epsilon$ : normalized difference between the experimental and the theoretical fields as defined in Eq. (9)).

Table 2

Peclet number $P e=\frac{U_{s} D_{d}}{a_{l}}$ averaged between 0 and $10 \mathrm{~ms}$.

\begin{tabular}{lrl}
\hline & Case 1 & Case 2 \\
\hline Isolated droplet & 209 & 578 \\
Monodisperse stream with Marangoni & 34 & 140 \\
Monodisperse stream without Marangoni & 60 & 231 \\
\hline
\end{tabular}

combusting droplets [19]. The observations suggest a larger influence of the internal convection on the heat transfer compared to the first case, where heat diffusion clearly prevails. This will be confirmed later by the simulations and the calculation of the Peclet number (Table 2).

\subsection{Comparison between the experimental and theoretical temperature fields}

The conditions for which the droplet stream has been simulated correspond to those for which temperature measurements were obtained (Table 1). To compare experiments with numerical simulations, it is important to apply on the simulation results the same spatial filtering than that resulting from the optical system on the measurements due to the size of the probe volume. Geometrical optics and ray tracing are used to determine theoretically the fluorescence signal that would have resulted from the temperature distribution obtained by DNS. Theoretical temperature maps are created from the reconstructed fluorescence signal, exactly in the same manner as described in Section 2. Additional information about this procedure is available in [15].

Naturally, theoretical maps share some features with their experimental counterparts. For instance, as it can be seen in Figs. $5 \mathrm{~b}$ and $6 \mathrm{~b}$, the area close to the surface is missing and that there is a low assymmetry between the left and the right of the temperature maps. Indeed, the fluorescence collection axis corresponds to the $x$ axis (as portrayed in Fig. 3) and the detection probe is located in the $x<0$ region in the reference frame of Figs. 5 and 6 . Thus, the focussing of the rays by the spherical interface of the droplet results in a measurement volume that is smaller on the right side $(x>0)$ of the temperature maps (keeping in mind that the measurement volume corresponds to the intersection between the droplets, the laser beams and the solid angle of the detection). As a result, spatial filtering by the measurement volume is more important on the left side of the temperature maps.

A parameter $\epsilon$ is introduced to evaluate the difference between the experimental and the theoretical fields, noted respectively $\xi_{\text {exp }}$ and $\xi_{\text {theo }}$ (Eq. (9)). In this expression, $v_{d}$ is the part of the droplet volume where the temperature is known. It excludes the region near the surface where the temperature cannot be determined experimentally. $\xi$ is the normalized temperature given by Eq. (10), where $T_{\min }$ and $T_{\max }$ are the minimum and maximum values of the temperature in the map of interest.

$$
\begin{aligned}
\epsilon & =\frac{1}{v_{d}} \times \iiint_{v_{d}}\left\|\xi_{\text {theo }}-\xi_{\text {exp }}\right\| d v \\
\xi & =\frac{T-T_{\min }}{T_{\max }-T_{\min }}
\end{aligned}
$$

Otherwise, the droplet passage into the igniter device is not considered in the calculations. Instead, the simulation starts with a uniform temperature inside the droplet. This temperature is equal to the temperature measured in the droplet center at the first measurement point located $2 \mathrm{~mm}$ above the igniter, not necessary equal to the injection temperature given in Table 1 due to the possible droplet change of temperature in the igniter. The time where the difference $\epsilon$ between the simulated and the first experimental temperature fields is the lowest is taken as the origin of time for the comparison between the simulation and experimental results in the Figs. 5 and 6.

After the simulations, the vapor flow rate is integrated to evaluate the mass variation of the droplets. This calculation results in a rather small decrease in the droplet mass during the time period of the simulation (Table 1 ) which justifies retroactively the hypothesis of a constant diameter. Additionally, due to the moderate velocity of the droplets, the Weber numbers are low for both cases (Table 1), justifying also the assumed spherical shape of the droplet.

The theoretical temperature maps calculated by the simulation are presented in Fig. 5b and Fig. 6b. It can be noticed that they compare well with the experimental measurements. As in the experimental results, the simulations predict that the isotherms 
close up on a cold area for case 1 and that the temperature is colder near the leading edge for case 2 .

The Peclet number $P e=\frac{U_{S} D_{d}}{a_{l}}$, expressing the ratio of heat convection to thermal diffusion, has been calculated from the simulation results as shown in Table 2 . The reference velocity chosen for the calculation of $P e$ is the maximum velocity at the droplet surface, i.e. around $90^{\circ}$ from the droplet axis of movement. The Peclet numbers indicated in Table 2 are averaged over the same period of time as the measurements (i.e. $10 \mathrm{~ms}$ ). The Peclet number is much lower in case $1(P e=34)$ than in case $2(P e=140)$ which confirms the observations made from the experiments.

However, there are also some differences between the experimental and simulation results. For instance, heat seems to be transported faster from the trailing edge to the leading edge in case 2 (Fig. 6b), while the cold zone remaining in the core of the droplet is slightly off-center in the simulation of case 1 (Fig. 5b). These differences suggest an overestimation of the internal circulation velocity but they remain small when compared to the results obtained without considering the Marangoni effect and the droplet interactions (see Sections 4.3 and 4.4).

\subsection{Analysis of the repartition of the forces and influence of the Marangoni force on the heat convection}

The transient evolution of the forces exerted on the droplet surface (including the friction drag $F_{\text {friction, }}$, the form $\operatorname{drag} F_{\text {form }}$ and the Marangoni force $F_{\text {Marangoni }}$ ) are depicted in Fig. 7 for the cases 1 and

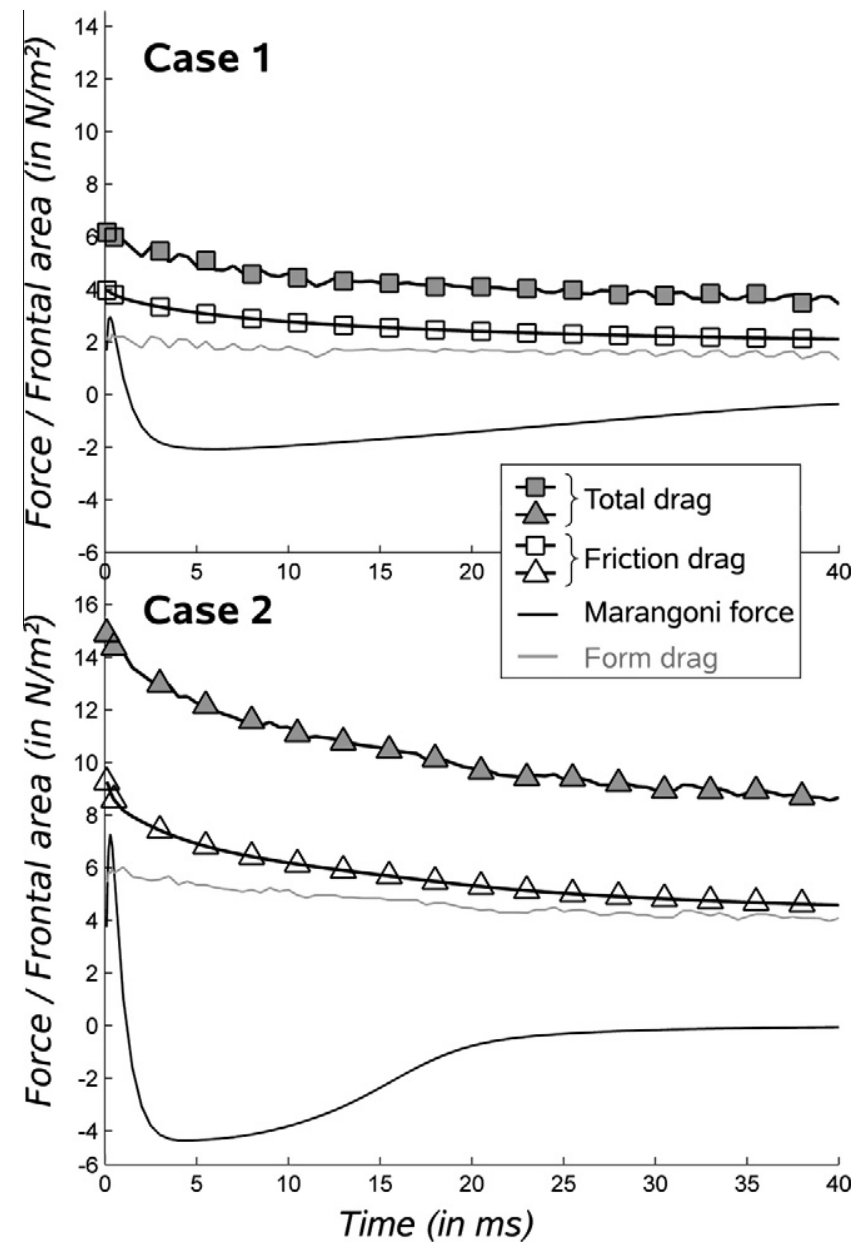

Fig. 7. Transient evolution of the forces exerted on the droplet surface for the cases 1 and 2 in Table 1 . Note that these forces are divided by the frontal area of the droplet and that the Marangoni force is not accounted in the total drag.
2. As mentioned in Section 3, the Marangoni force is not accounted in the total drag $F_{d r a g}$. Otherwise, all the presented forces are divided by the droplet frontal area for commodity reasons. A first analysis reveals that the friction and form drag have a comparable contribution, even if the friction drag is for both cases higher. Moreover, they present similar decreases with time, due to the increase of the vapor flow rate, i.e. the surface temperature becomes hotter; hence the blowing effect induced by the Stefan flow increases [23]. The drag force appears to be larger for case 2 . This is mainly due to the fact that the droplet velocity is several $\mathrm{m} / \mathrm{s}$ higher for case 2 and the drag force increases with the square of the droplet velocity.

As stated before, the simulations were performed neglecting the thrust force due the vapor ejection at the droplet surface. A justification can be given after the calculations. The thrust force, divided by the frontal area, can be roughly estimated from the ejection velocity $v_{e}$ and the total flow rate $q_{m}$ at the droplet surface (Eq. (11)).

$F_{\text {thrust }}=\frac{1}{\pi r_{S}^{2}} v_{e} q_{m}=\frac{1}{\pi r_{S}^{2}} \frac{\dot{m}}{\rho} \dot{m} 4 \pi r_{S}^{2}=\frac{4 \dot{m}^{2}}{\rho}$

Eq. (11) yields values inferior to $0.2 \mathrm{~N} / \mathrm{m}^{2}$ for the droplet in the monodisperse streams, and inferior to $1.2 \mathrm{~N} / \mathrm{m}^{2}$ for the isolated droplet, after $40 \mathrm{~ms}$ of evaporation (i.e. after the end of the heating period when evaporation occurs almost at a constant rate). The difference between the isolated droplet and the monodisperse stream is due to the wake effect in the stream, which saturates the vicinity of the leading edge in vapor; hence reducing strongly the vapor flow rate. Nevertheless, this calculation largely overestimates the thrust force since it considers that the entire vapor is ejected in the same direction. In fact, the direction of the vapor ejection must be balanced between the leading edge and the trailing edge, by integrating this thrust force expressed locally over the whole surface. Moreover, due to the axi-symmetry, only the contribution of the force component in the direction of the droplet motion should be accounted for in the integration. A careful calculation of this integral leads to lower values of the thrust force about $0.5 \mathrm{~N} / \mathrm{m}^{2}$ for an isolated droplet and $0.05 \mathrm{~N} / \mathrm{m}^{2}$ for a droplet in a monodisperse stream at $40 \mathrm{~ms}$. In comparison, the drag force is about $30 \mathrm{~N} / \mathrm{m}^{2}$ for the isolated droplet and $10 \mathrm{~N} / \mathrm{m}^{2}$ for the droplet in a monodisperse stream. It is consequently acceptable to neglect the thrust force in the present investigation, even with the isolated droplet case.

Fig. 7 also shows that the Marangoni force has a significant contribution to the stress at the droplet surface. As already noticed by Dwyer and co-workers [6], the evolution of the Marangoni force can be divided in two periods:

- An initial period of a few ms where the Marangoni effect accelerates the liquid motion since the Marangoni force has a positive sign. After the initial condition, where a uniform temperature is imposed, the surface temperature increases more rapidly near the leading edge due to transfer parameters that are higher in this area. A higher temperature at the leading edge corresponds to a positive Marangoni force whose contribution adds to the friction drag [6]

- A second period where the Marangoni effect slows down the liquid motion, the Marangoni force having a negative sign. The hotter liquid is transported by the internal circulation from the leading edge to trailing edge, which leads to an inversion of the surface temperature, the area near the trailing edge becoming warmer. This period is longer, and the negative Marangoni force presents a comparable contribution as the friction drag. At the end of this period, the Marangoni force tends to 0 as the droplet reaches a uniform temperature at the end of its heating. 


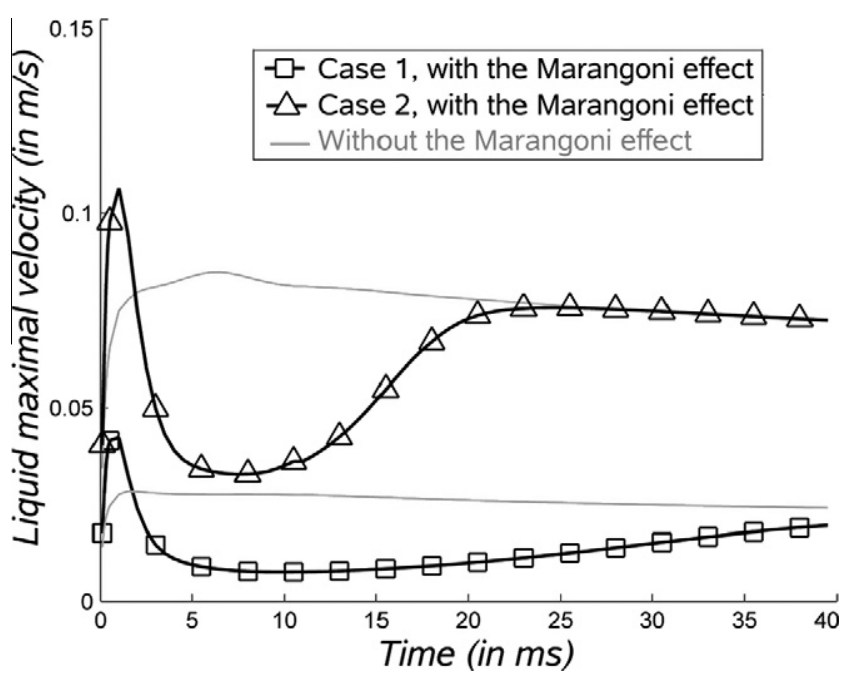

Fig. 8. Influence of the Marangoni effect on the liquid maximal velocity for the cases 1 and 2 in Table 1.

To highlight the influence of the Marangoni effect on the internal convection, the droplet heating was also simulated without accounting for the Marangoni effect. Fig. 8 shows the evolution of the maximum liquid velocity with and without the Marangoni effect for cases 1 and 2 . In the absence of the Marangoni force, the liquid maximal velocity reaches in a few ms a quasisteady state. The evolution of the maximum liquid velocity is mainly driven by the slow decrease of the friction drag during the evaporation. In presence of the Marangoni force, the previously mentioned accelerating and decelerating periods are clearly visible. The variations in the liquid velocity due to the Marangoni effect are rather important. The maximum liquid velocity can be reduced by nearly $50 \%$ during the deceleration period compared to the case without Marangoni effect. The Peclet numbers (Table 2 ) averaged without the Marangoni effect are consequently higher for both cases ( $P e=60$ for case 1 and $P e=231$ for case 2 ). In agreement with the evolution of the Marangoni force (Fig. 7), at the end of the droplet heating, the liquid velocity increases again up to the same value as that obtained without the Marangoni effect.

As it can be observed in Figs. 7 and 8, the decelerating period lasts much longer for case 1 than for case 2 . Although case 2 exhibits a stronger Marangoni force (Fig. 7), in terms of relative values this force is in fact weaker (Table 3). As a result of this weaker relative Marangoni force and the higher friction drag, the internal circulation is enhanced in case 2 . Therefore a homogeneous temperature is reached more rapidly causing the diminishing of the Marangoni force.

Fig. 5c and Fig. $6 c$ present the evolution of temperature field in the droplet in the absence of Marangoni effect for both cases. A very clear change in the temperature distribution within the droplet can be observed after a few ms. In the absence of Marangoni effect, advection seems to play a more important role: heat is transported more rapidly from the trailing edge to the droplet

Table 3

Relative contribution of the friction drag, form drag and Marangoni force between 10 and $40 \mathrm{~ms}$ (values are determined by dividing the forces by the total drag).

\begin{tabular}{lllll}
\hline & Case 1 & \multicolumn{3}{c}{ Case 2} \\
\hline Spacing parameter & Isolated & $C=2.3$ & Isolated & $C=4.0$ \\
Viscous drag & $+50 \%$ & $+60 \%$ & $+47 \%$ & $+53 \%$ \\
Form drag & $+50 \%$ & $+40 \%$ & $+53 \%$ & $+47 \%$ \\
Max Marangoni force & $+40 \%$ & $+50 \%$ & $+30 \%$ & $+50 \%$ \\
Min Marangoni force & $-30 \%$ & $-45 \%$ & $-20 \%$ & $-36 \%$ \\
\hline
\end{tabular}

center and the leading edge. This evolution is consistent with previous remarks. During most of the time (the decelerating period), the Marangoni force slows the liquid motion in the droplet. The accelerating period is indeed too short to have a noticeable impact on the temperature fields. As expected, the differences between the simulations and the temperature measurements (Fig. 5a and Fig. 6a) are more pronounced when the Marangoni effect is not taken into account. This appears clearly in the values of $\epsilon$, which are much higher in the absence of Marangoni effect and confirms the importance of the Marangoni effect in the description of the droplet heating.

Finally, the differences mentioned at the end of Section 4.2 between the experimental maps and the theoretical maps with the Marangoni effect can be interpreted as a consequence of the initial condition. Without considering the ignition, the simulation starts with a uniform temperature, inducing an accelerating Marangoni effect in the first initial period of heating. However, in the experiments, the igniter already induces thermal gradients at the droplet surface. Therefore, the droplets are probably in their decelerating second period when the comparison with the simulation starts, which is not taken into account with this approach.

\subsection{Influence of the droplet interaction}

In order to highlight the effect of aerodynamic interactions on the droplet heating, the case of an isolated droplet has been also simulated, including the Marangoni effect. Simulations of the isolated droplet are performed using the same initial and ambient conditions as the corresponding interacting droplets (Table 1).

Fig. 9a and Fig. 9b shows the evolution of the temperature field of the isolated droplets that were predicted by the simulation. As expected, the heating of the isolated droplet is much faster and the temperature tends to become uniform more rapidly due to a stronger internal convection. The internal convection is so important for the isolated droplet that isotherms quickly coincide with the streamlines of the internal flow, which are drawn on the right side of the temperature maps.

An analysis of the effect of droplet interactions on the drag can be based on the form and viscous drag divided by the total drag, presented in Table 3. On the one hand, the cases with droplets in interaction $(C=2.3$ and $C=4)$ can be compared. It appears that the form drag has a weaker contribution to the total drag force when $C=2.3$. On the other hand, considering the isolated droplets, form and viscous drag have almost the same contribution to the drag force. This preliminary remark emphasizes that the interactions modify the balance between the form and viscous drag in a much more important way than the velocity and the diameter of the droplets.

Fig. 10 shows the time evolution of the ratios $\frac{C d}{C d_{i s o}}$ and $\frac{C_{f}}{C_{f, i s o}}$ for cases 1 and 2 . It appears that the spacing parameter has a great influence on the overall drag force and its viscous drag component. For the drag coefficient, this behavior has been already pointed out by Atthasit et al. [14], who measured the droplet deceleration for different spacing parameters in an ethanol monodisperse droplet stream, leading to a corrective factor. Presently, this correction predicts a ratio equals to $13 \%$ and $29 \%$, respectively for cases 1 and 2 . These values are slightly lower than those observed in Fig. 10 but the tendency is correct. It should be mentioned that the drag coefficient $C d_{i s o}$ in the correction of Atthasit et al. corresponds to the drag coefficient of an isolated droplet that would have experienced exactly the same velocity and temperature as the corresponding interacting droplet. This differs from the calculation used to obtain Fig. 10, in which the isolated droplet evolves freely from the same initial condition as the interacting droplet.

The ratio $\frac{C_{f}}{C_{f, i s o}}$ can be compared to the results of Castanet et al. [19]. The latter found a value of about $16 \%$ in the case of ethanol 

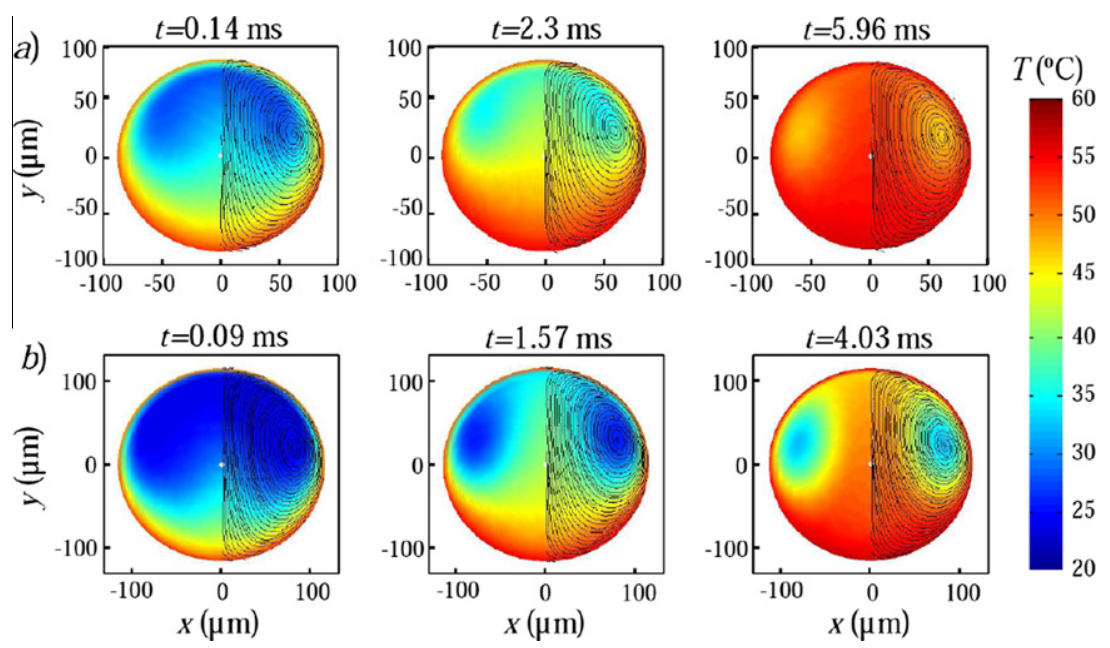

Fig. 9. Evolution of the temperature distribution in a meridian plane within isolated droplets corresponding to the conditions given in Table 1 ((a) case 1 , (b) case 2).

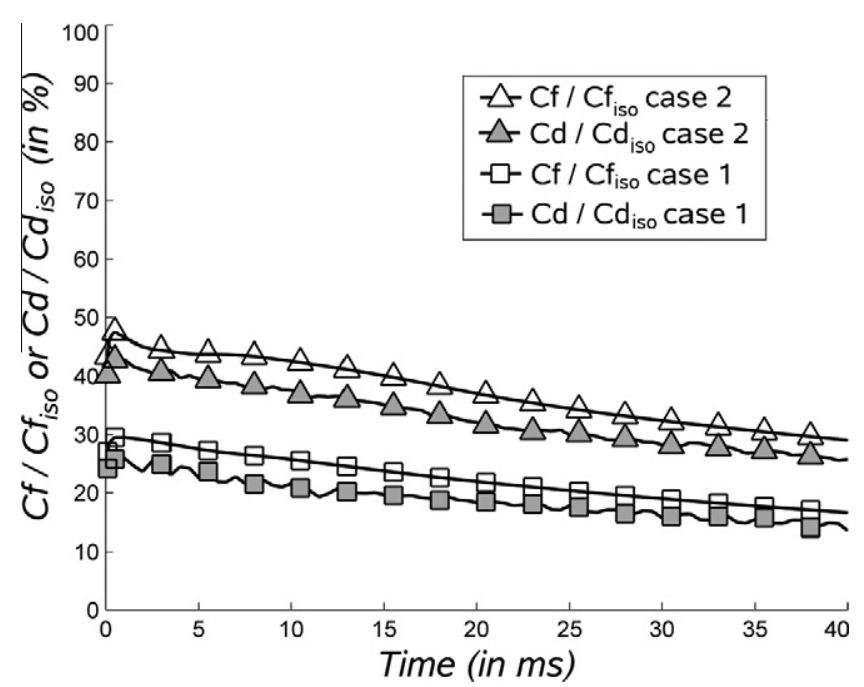

Fig. 10. Evolution of the ratios $\frac{C d}{C d_{\text {iso }}}$ and $\frac{C_{f}}{C_{f, i s o}}$ within the droplet stream corresponding to the cases 1 and 2 in Table 1.

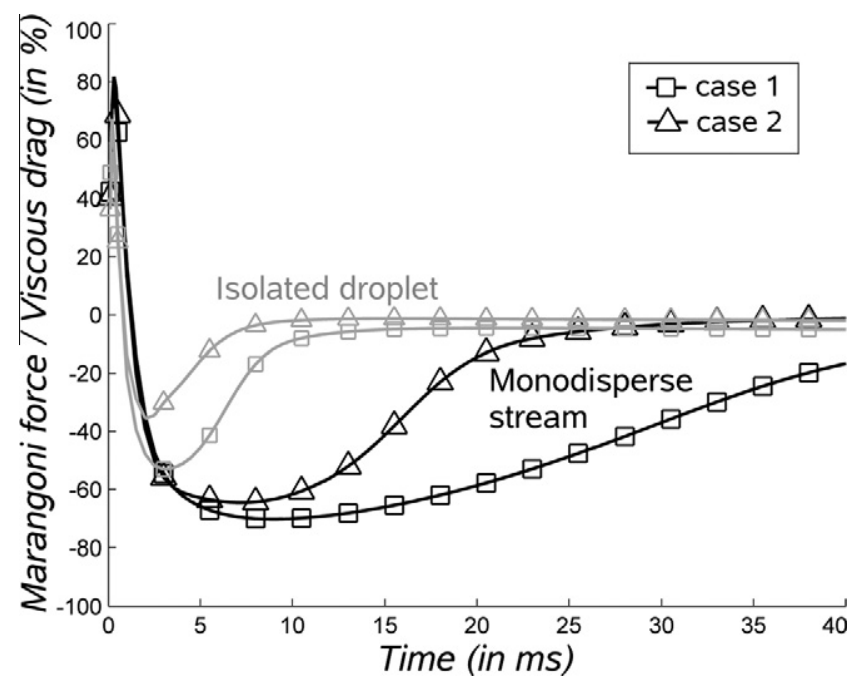

Fig. 11. Relative contribution of the Marangoni force compared to the viscous drag for the cases 1 and 2 in Table 1, within the isolated droplet and the monodisperse stream. combusting droplets with a spacing parameter averaging 4 . This value is much lower than observed in the present simulation $\left(\frac{C_{f}}{C_{f}}\right.$ is about $40 \%$ for case 2 ). However, the determination of $C_{f}$ by Castanet et al. [19] was made on the basis of a comparison between the experimental temperature fields and simulations with the Hill vortex model, i.e. without accounting for the Marangoni effect. As a result, they gave to $C_{f}$ a lower value that includes the negative contribution of the Marangoni force. Because the absolute value of the Marangoni force in the case 2 is about two times smaller than that of the friction drag (during the first $10 \mathrm{~ms}$ of the droplet heating considered in [19]), the simulation results obtained in this study are in fact rather consistent with the results of Castanet et al. [19].

In Fig. 10, the ratio $\frac{C d}{C d_{\text {iso }}}$ is lower than the ratio $\frac{C_{f}}{C_{f \text { iso }}}$ for both cases, which infers a more significant effect of the droplet interactions on the form drag than on the friction drag. This behavior results from the wake effect influencing the local pressure and velocity distribution around each droplet in the stream. The friction drag would be less affected by the interactions since its maximal value is located between the leading edge and the trailing edge in a region of the surface that is less exposed to the wake of the previous droplet. This justifies also the weaker contribution of the form drag observed in the evolution of the surface forces (Fig. 7, Table 3) when the droplets are in a very strong interaction regime, i.e. in case 1 .

Finally, the relative contribution of the Marangoni force compared to the viscous drag is depicted in Fig. 11, for the cases of the isolated droplets and the monodisperse droplet streams. It appears that the droplet interactions result in a longer and stronger Marangoni effect during the decelerating regime, as underlines the case 1 with the lowest spacing parameter. Without interactions, the larger friction on the droplet surface enhances dramatically the liquid motion and reduce the relative contribution of the Marangoni effect in terms of duration and relative intensity.

\section{Conclusion}

This study is based on advanced complementary experimental and numerical techniques to investigate the heating of evaporating droplets in regime of aerodynamic interactions. A very detailed numerical simulation takes into account most of the physical aspects driving the droplet heating and especially the heat transport by the internal circulation. It includes the forced convection of the gas phase, the wake effects behind the leading droplets, the nonuniform heat, mass and momentum transfers at the droplet surface, the liquid motion induced by the liquid-gas frictions and 
the Marangoni stress due to thermo-capillary effects. The measurements of the temperature field within the droplet using the 2-color LIF thermometry validate the results obtained by the simulation. This comparison emphasizes the predominant role of the Marangoni stress which slows down dramatically the liquid flow velocity during the droplet heating period. By decreasing the Peclet number, the Marangoni effect modifies the whole temperature distribution within the droplet. Results reveal that only simulations accounting for the Marangoni effect can properly describe the temperature within ethanol droplets in strong interactions, where the friction on the droplet surface, in addition to the heating and vaporization rates, are sharply reduced. The balance between the friction and form drag is modified and the relative contribution of the Marangoni stress is enhanced.

A comprehensive survey of the physical effects that may influence the droplet heating in a regime of strong interaction is provided by this analysis. The results have been obtained for a simplified configuration, but they describe in a quantitative manner the evaporation of droplets in dense sprays in overheated atmospheres. Finally, this study underlines the benefits of analyzing the flow physics in a fundamental configuration, like the monodisperse droplet stream, in order to improve models used to simulate industrial sprays. Nevertheless, the size of the droplets considered in this study is large in comparison with the droplet sizes in a conventional IC engine. For smaller droplets, the velocity of the internal circulation flow decreases when only the drag force (excluding the Marangoni force) is considered. At the same time, the substantially higher injection velocity in an IC engine is expected to maintain the internal convection at a significant level rather comparable in its effects to the situations studied in this paper. Moreover, when decreasing the droplet size, the Marangoni stress increases to a larger extent than the other surface stresses, i.e. the friction and form drag. This amplifies the importance of the Marangoni effect for these small droplets.

\section{Acknowledgments}

The numerical part of this work has been supported by the French environmental agency (ADEME) and the car manufacturer Renault. Further improvements have been carried out with support of the Deutsche Forschungsgemeinschaft (GRK 1114). The experimental part has been performed in the framework of the ASTRA program, supported by the French CNRS and ONERA.

\section{References}

[1] W.E. Ranz, W.R. Marshall, Evaporation from drops, Chem. Eng. Progr. 48 (1952) 141-146. 173-180.
[2] G.A.E. Godsave, Studies of the combustion of drops in a fuel spray: the burning of single drops of fuel, in: Proceedings of the 4th International Symposium on Combustion, Williams and Wilkins, Baltimore, 1953, pp. 818-830.

[3] S. Prakash, W.A. Sirignano, Liquid fuel droplet heating with internal circulation, Int. J. Heat Mass Transfer 21 (1978) 885-895.

[4] M. Renksizbulut, R.J. Haywood, Transient droplet evaporation with variable properties and internal circulation at intermediate Reynolds numbers, Int. J. Multiphase Flow 14 (1988) 189-202.

[5] B. Abramzon, W.A. Sirignano, Droplet vaporization model for spray combustion calculations, Int. J. Heat Mass Transfer 32 (1989) 1605-1618.

[6] H. Niazmand, B.D. Shaw, H.A. Dwyer, I. Aharon, Effects of Marangoni convection on transient droplet evaporation, Comb. Sci. Technol. 103 (1994) 219-233.

[7] M. Labowsky, Calculation of the burning rates of interacting fuel droplets, Comb. Sci. Technol. 22 (1980) 217-226.

[8] M. Marberry, A.K. Ray, K. Leung, Effect of multiple particle interactions on burning droplets, Comb. Flame 57 (1984) 237-245.

[9] T.A. Brzustowski, E.M. Twardus, S. Wojcicki, A. Sobiesiak, Interaction of two burning fuel droplets of arbitrary size, AIAA J. 17 (1979) 1234-1242.

[10] C.H. Chiang, W.A. Sirignano, Interacting, convecting, vaporizing fuel droplets with variable properties, Int. J. Heat Mass Transfer 36 (1993) 875-886.

[11] C.H. Chiang, W.A. Sirignano, Axisymmetric calculation of three droplet interaction, Atom. Sprays 3 (1993) 91-107.

[12] J.J. Sangiovanni, M. Labowsky, Burning times of linear fuel droplet arrays: a comparison of experiment and theory, Comb. Flame 45 (1982) 15-30.

[13] J.F. Virepinte, P. Magre, G. Collin, G. Lavergne, Y. Biscos, A rectilinear droplet stream in combustion: droplet and gas phase properties, Comb. Sci. Technol. 150 (2000) 143-159.

[14] A. Atthasit, N. Doue, Y. Biscos, G. Lavergne, Influence of droplet concentration on the dynamics and evaporation of a monodisperse stream of droplets in evaporation regime, in: Proceedings of 1st International Symposium on Combustion and Atmospheric Pollution, St. Petersburg, Russia, 2003.

[15] G. Castanet, P. Lavieille, M. Lebouché, F. Lemoine, Measurement of the temperature distribution within monodisperse combusting droplets in linear stream using two colors laser induced fluorescence, Exp. Fluids 35 (2003) 563571.

[16] V. Deprédurand, P. Miron, A. Labergue, M. Wolff, G. Castanet, F. Lemoine, A temperature-sensitive tracer suitable for two-colour laser-induced fluorescence thermometry applied to evaporating fuel droplets, Meas. Sci. Technol. 19 (2008) 105-403.

[17] G. Castanet, A. Delconte, F. Lemoine, L. Méès, G. Gréhan, Evaluation of temperature gradients within combusting droplets in linear stream using two colors laser-induced fluorescence, Exp. Fluids 39 (2005) 431440.

[18] G. Castanet, M. Lebouché, F. Lemoine, Heat and mass transfer of combusting monodisperse droplets in a linear stream, Int. J. Heat Mass Transfer 48 (2005) 3261-3275.

[19] G. Castanet, F. Lemoine, Heat transfer within combusting droplets, Proc. Combust. Inst. 31 (2007) 2141-2148.

[20] C. Maqua, G. Castanet, F. Grisch, F. Lemoine, T. Kristyadi, S.S. Sazhin Monodisperse droplet heating and evaporation: Experimental study and modelling, Int. J. Heat Mass Transfer 51 (2008) 3932-3945.

[21] B. Frackowiak, A. Strzelecki, G. Lavergne, A liquid-vapor interface positioning method applied to PLIF measurements around evaporating monodisperse droplet streams, Exp. Fluids 46 (2009) 671-682.

[22] A. Labergue, V. Deprédurand, A. Delconte, G. Castanet, F. Lemoine, New insight into two-color LIF thermometry applied to temperature measurements of droplets, Exp. Fluids 49 (2010) 547-556.

[23] B. Frackowiak, G. Lavergne, C. Tropea, A. Strzelecki, Numerical analysis of the interactions between evaporating droplets in a monodisperse stream, Int. J. Heat Mass Transfer 53 (2010) 1392-1401.

[24] Exciton Inc. Copyright. <http://www.exciton.com>

[25] OpenFOAM, OpenCFD Ltd. <http://www.opencfd.co.uk/openfoam>. 